\title{
Clinical Implications in Understanding Prostate Cancer Biology: From Androgen Receptor to Molecular Target Therapy in Metastatic Castration Resistant Prostate Cancer
}

\section{Eliza Dalsasso Ricardo*, Angel AT Uchiyama, Denis S Shimba and Ariel G Kann}

Department of Clinical Oncology, Hospital Alemao Oswaldo Cruz, Brazil

*Corresponding Author: Eliza Dalsasso Ricardo, Department of Clinical Oncology, Hospital Alemao Oswaldo Cruz, Brazil.
Received: April 07, 2021

Published: May 15, 2021

(C) All rights are reserved by Eliza Dalsasso

Ricardo., et al.

\begin{abstract}
Prostate cancer is one of the most common malignancies in the world. For a long time, the cornerstone of treatment for advanced disease has been the manipulation of hormonal pathway of testosterone since is well stablished that androgen receptor regulates prostate growth, differentiation and apoptosis in regular and cancer cells. However, during the treatment of metastatic disease with androgen deprivation therapy resistance mechanisms appear, which led to the development of new treatments, such as secondgeneration anti-androgens-like (such as enzalutamide, apalutamide and darolutamide), and abiraterone acetate. In addition to the development of this new antiandrogens the deeper knowledge about DNA damage repair and their pathways such as direct reversal, mismatch repair, base excision repair, homologous recombination and the non-homologous end joining, resulted in new opportunities of treatment. Recently, PARP inhibitors have showed their efficacy in the treatment of advanced prostate cancer. Therefore, the aim of this article is to review the current knowledge about cancer prostate biology and its clinical implications.
\end{abstract}

Keywords: Prostate Cancer; Biology; Androgen-receptor; Molecular Therapy; DNA Damage Repair; PARP Inhibitor

\section{Abbreviations}

PCa: Prostate Cancer; DHT: Dihydrotestosterone; ADT: Androgen Deprivation Therapy; AR: Androgen Receptor; T: Testosterone; GnRH: Gonadotropin-releasing Hormone; GnRH-R: Gonadotropin-releasing Hormone Receptor; LH: Lutinizing Hormone; FSH: Follicle Stimulating Hormone; DHEA: Dehydroepiandrosterone; 3 $\beta$-HSD: $3 \beta$-Hydroxysteroid Dehydrogenase; CRPC: Castration Resistant PC; PARP: (polyADP-ribose) Polymerase; DDR: DNA Damage Repair; DSB: Double Strand Breaks; HRD: HR Deficiency; LOH: Loss of Heterozygosity; DDRe: DNA Damage Response; mCRPC: Metastatic Castration-resistant PC; PTEN: Phosphatase and Tensin Homolog; RAC-alpha serine/threonine-protein Kinase AKT; mTOR: Mammalian Target of Rapamycin; PARP: Inhibitor iPARP

\section{Introduction}

Prostate cancer is one of the 5 most common malignancy and according to GLOBOCAN is expected that $1,414,259$ new cases of prostate cancer will occur in the world in 2020, corresponding to $7.3 \%$ of all neoplasms diagnoses and 375,304 deaths [1]. It is known that most patients present at diagnosis as localized or regional disease $(89 \%)$ and in general the 5-year survival rate is $97.8 \%$. However, when disease is metastatic, the 5-year survival rate is only $30 \%$ [2]. The most important risk factor for the development of prostate cancer is aging. Epidemiologic studies have shown that the risk of prostate cancer is higher in African American compared with other ethnic groups and that it occurs at an earlier age [3]. Genetic factors, especially germline mutations in DNA repair genes appear to play a role in the development of certain prostate cancer and maybe associated with more aggressive disease [4]. Other factors, such as diet, hormone levels and obesity have been studied, but their role appears limited [3].

For a long time, the main systemic treatment for advanced disease has been the manipulation of hormonal pathway of testoster- 
one. Since seminal Huggins's study, understanding the testosterone pathway as primarily responsible for the progression of prostate cancer has led researching and treatment of prostate cancer [5]. Only in 2004 with the publication of the TAX 327 trial, cytotoxic chemotherapy became an option in the therapeutic arsenal. In this trial of patients with castration-resistant metastatic prostate cancer, the use of docetaxel resulted in $24 \%$ reduction in the risk of death, but eventually chemotherapy fails, and therapeutic options are needed [6].

Fortunately, in the last decade the better understanding of the testosterone pathway and its relationship with the androgen receptor as well the steroidogenesis in the adrenal gland has promoted the development of new therapies, the so-called novel antiandrogen therapy, like abiraterone, enzalutamide, apalutamide and darolutamde [7-10]. In addition to that, the knowledge that molecular routes like DNA repair pathway are also responsible for tumor growth has led to the emergence of new horizon of therapeutic options [4]. Deficiency in the DNA repair pathway is one of the hallmarks in the development of cancer as it causes genomic instability, and the development of molecular target therapies is currently a revolution in the treatment of prostate cancer. The purpose of this article is to review the biology of prostate cancer and its relationship with hormonal and molecular pathways.

Biology of prostate cancer

Androgenic pathway

Prostate cancer ( $\mathrm{PCa}$ ) has been recognized as a hormone-dependent affection since early 1940s. Experimental demonstrations observed tumor regression after reducing serum androgen concentrations by orchiectomy or exogenous estrogen administration, and tumor activation by androgen injections [4] Hormone modulation of PCa growth provides the basis for androgen deprivation therapy (ADT), which is the core treatment of advanced PCa [11]. Its primary objective is to reduce circulating levels of androgens. The two principal androgens in men are testosterone, produced by testicular Leydig cells, and dihydrotestosterone (DHT), produced from testosterone in peripheral tissues by 5 - $\alpha$ reductase [12].

The prostate contains high affinity androgen receptor (AR) cells that regulate prostate growth, differentiation and apoptosis in regular and cancer cells by promoting and inhibiting secretion of various growth factors from stromal cells that act on the epithelial ones [13]. Androgens, such as testosterone (T) and dihydrotestosterone (DHT), are the most important agonists for AR. DHT has a 10-fold higher affinity for the androgen receptor and thus it is the primary ligand for the AR in the prostate [14]. When androgens bind to the
$\mathrm{AR}$, it induces conformational change and subsequent translocation of the AR into the nucleus. Nuclear AR binds to androgen responsive elements in the DNA, resulting in transcriptional activity that can induce cellular proliferation and cellular differentiation [15].

Gonadal steroid hormone production is stimulated by the hypothalamic-pituitary-axis through synthesis and release of Gonadotropin-releasing hormone (GnRH), also referred to as LHRH. This peptide is produced by a small number of hypothalamic neurons, which are released in a pulsatile way into the hypophyseal circulation to reach the gonadotrophs in the anterior pituitary where it binds to its specific receptors (GnRH-R). By binding to these receptors, GnRH triggers the synthesis and release of the two gonadotropins LH (luteinizing hormone) and FSH (follicle stimulating hormone), which are responsible for inducing the production of testosterone in the testes [16] as seen in figure 1.

Another pathway to steroid biosynthesis happens through a complex mechanism that takes place in the cortex of the adrenal gland and in the genital tissue [17]. The process starts with the conversion of cholesterol to pregnenolone. This conversion is done by the mitochondrial cholesterol side-chain cleavage enzyme (cytochrome P450scc) encoded by the cholesterol side-chain cleavage gene (CYP11A1) [18]. Then, pregnenolone is converted to progesterone by $3 \beta$-hydroxysteroid dehydrogenase ( $3 \beta$-HSD) These two metabolites are the precursors for others steroid hormones $[19,20]$. Pregnenolone and progesterone are hydroxylated by CYP17 (an enzyme with both $17 \alpha$-hydroxylase and 17,20-lyase activities) to form $17 \alpha$-hydroxysteroids [18]. From there on, the 17,20 -lyase activity converts the two $17 \alpha$-hydroxylated steroids to dehydroepiandrosterone (DHEA) and androstenedione.

Finally, in the zona reticularis of the adrenal gland, 17ßHSD5 (encoded by AKR1C3) converts small amounts of androstenedione to testosterone, whereas in the testis this reaction is catalyzed by $17 \beta$ HSD [18] as seen in figure 1.

Chronic administration of LHRH agonists results in suppression of the hypothalamic-pituitary-gonadal axis by downregulation of the pituitary receptors for LHRH, leading to suppression of folliclestimulating hormone (FSH) and LH. This reduces the production of testosterone in the testes resulting in castrate levels of testosterone [21,22]. Many synthetic LHRH agonists (such as goserelin, leuprolide, buserelin and triptorelin) are now used as first-line hormonal treatment in patients with advanced-stage prostate cancer. However, the use of LHRH agonists is associated to a 'testosterone flare' 
phenomenon, an initial increase in testosterone production; this event can trigger exacerbation of pain, worsen obstructive urinary symptoms and in rare cases it can lead to ureteral obstruction or spinal cord compression. This 'testosterone flare' can be overcome by association with AR blocker-likes [23], and it has also led to the development of LHRH antagonists that directly bind to the LHRH receptors without causing the initial testosterone surge. Currently, degarelix is an LHRH antagonist that is well established for clinical use [24]. In December 2020, the Food and Drug Administration approved the first oral gonadotropin-releasing hormone ( $\mathrm{GnRH}$ ) receptor antagonist, relugolix. Based on HERO (NCT03085095), a phase 3 open label trial which showed that $96.7 \%$ men achieved sustained testosterone suppression to castrate levels through 48 weeks of treatment [25]. Relugolix is a new treatment perspective because it leads to a rapid testosterone suppression without an initial testosterone surge and its facility in administration.

Additionally, antiandrogens therapy is also used in metastatic or recurrent PCa. Antiandrogen molecules prevent binding of androgen to the AR in the cytoplasm. This inhibits the AR signaling pathway and thus prevents translocation of the AR into the nucleus and binding to DNA, decreasing cell proliferation and inducing cell death [26]. First generation antiandrogens (like flutamide, bicalutamide and nilutamide) can be used in association with LHRH agonist and antagonist in order to achieve "complete androgen blockade" [27].

In some cases, PCa cells develop alternative mechanisms of AR signaling, AR amplification, alternative splicing, intratumoral androgen production, or adrenal gland testosterone production. In these cases, the treatments aforementioned lose their efficacy and the disease is termed castration resistant PC (CRPC) [28]. Also, an adaptive response to androgen deprivation has been observed in CRPC where there were intratumoral testosterone production and $5 \alpha$-DHT biosynthesis, which lead to the understanding that ADT alone is insufficient to reduce androgens precursors [7]. The need to overcome these resistance mechanism has led to the development of new treatments, such as second-generation anti-androgens-like (such as enzalutamide, apalutamide and darolutamide), and abiraterone acetate, which is a CYP17A1 inhibitor that targets both 17a-hydroxylase and 17,20-lyase activities and inhibits residual androgen biosynthesis [7]. These medications are currently largely used in the CRPC scenario in clinical practice.

Treatment response is often short-lived in metastatic CRPC because patients develop tumor resistance, and so novel improved therapeutic options are needed [29]. Studies have been exploring immunotherapy and inhibition of poly(ADP-ribose) polymerase (PARP) in tumors with DNA repair defects inducing synthetic lethality [30]; more randomized trials are required to test the interplay between androgen receptor signaling and PARP functions [31].

\section{DNA damage repair pathways}

The field of PCa has benefited from the emergence of multiple new therapies over the past few years and the best understanding about DNA damage repair (DDR) provided the use of some drugs in PCa [4].

The genome is constantly under the action of external and internal agents that cause DNA damage. Mutagenesis has an important role in the maintenance of the genome and evolution of the species, but it can contribute to the appearance of cancer. So, deleterious mutations due to DNA lesions must be repaired. Cells respond to DNA damage by instigating robust DNA damage response pathways. These pathways include the direct reversal pathway, the mismatch repair (MMR) pathway, the nucleotide excision repair (NER) pathway, the base excision repair (BER) pathway, the homologous recombination (HR) pathway, and the non-homologous end joining (NHEJ) pathway. This way, mechanisms that can be deregulated such as repair DNA, DNA damage tolerance and DDR pathways, which increases mutagenesis and genomic instability, can lead to cancer progression [32-34].

Researchers realized that a variety of currently known proteins have critical functions in maintaining DNA integrity, particularly regarding the recognition and repair of DNA damage through multiple pathways. Then, they have an important role in maintaining the genomic integrity of the cell and ensures its ability to persist and proliferate [35,36]. DDR deficiency plays an important role in genome instability and mutations in tumors and has been recognized as one of the hallmarks of cancer, and provides a logical framework for understanding the diversity of neoplastic diseases $[37,38]$. Mutations that modify the function of genes involved in repairing DNA damage through homologous recombination have been shown to be associated with the aggressive clinical behavior of PCa and with cancer-specific mortality. In addition, they contribute to destabilizing PC cells, often making them more susceptible to cell death $[36,39]$.

Homologous recombination (HR) has a fundamental role in carcinogenesis. HR pathway is essential for high-fidelity DNA double strand break repair. When defective HR occurs, it results in chromatid exchanges leading to genomic instability. Cells deficient in 
HR are known to be sensitive to ionizing radiation and chemotherapeutic drugs. That affects both strands of DNA and acts in the S/ G2-phases of the cell cycle where HR is the preferential pathway of double strand breaks (DSB) repair. HR pathway involves several genes, including BRCA1 and BRCA2. HR deficiency (HRD) due to inactivation of such genes leads to increased levels of genomic alterations. HRD causes loss of heterozygosity ( $\mathrm{LOH})$, telomeric allelic imbalance and large-scale state transitions. $\mathrm{LOH}$ results from one allele being lost from a cell that is then either homozygous or hemizygous for the remaining allele. In addition to that, the extent of genome wide $\mathrm{LOH}$ provides a single global assessment of HRD irrespective of causative lesion, and the potential for using it as a therapeutic target [40-42].

When compared to the general population, the risk of PC is estimated to be 1.8-3,75 fold higher for people with germline BRCA1 and 2,5-8,6 fold with germline BRCA2. Moreover, the data about the frequency of germline mutation of DDR in men (12\%) is higher than that in the general population. Thus, DDR deficiency becomes a potential PCa driver [43]. When evaluating men with BRCA2 mutations, it was found that they can cause a higher risk of developing life-threatening prostate cancer, and these mutations may be associated with worse treatment outcomes within the setting of castration-resistant disease. It is known that BRCA2 mutations are involved in the repair of-DSB through homologous recombination and are also important in several other processes central to maintaining genome stability, including DNA replication, telomere homeostasis and cell cycle progression [39,44]. Dall Era., et al. evaluated 152 patients harvested from metastatic and localized tumors that presented with germline or somatic mutation in $\geq 1$ DNA repair genes. The most prevalent mutations were BRCA2 (11.4\%) and ATM (5.8\%) and highest rates of DNA repair mutation were found in solid organ metastases including brain (36\%), liver (34\%), and lung (32\%) [39]. The role of DNA gene mutations in localized PCa was analyzed by Marshall., et al. In this trial, patients with Gleason grade group 3 and higher were 2.2 times more likely to harbor any DNA repair mutation and 2,7 times more likely to have BRCA1/2 or ATM mutations compared to those in Gleason grade groups 1-2. Moreover, the data about patients with pathologic T3 e T4 were 2,6 times more likely to have any DNA repair mutations and were 3,2 times more likely to have BRCA1/2 or ATM mutations compared to those with pT2 disease [45]. The analyses about prevalence of DNA damage response (DDRe) gene mutation in PCa was carried out by Lang., et al. Median prevalence rates for DDRe germline mutation were, $18.6 \%$ in PCa, $11.6 \%$ in metastatic PCa and $8.3 \%$ in metastatic castration-resistant PC (mCRPC). Median prevalence rates for
DDRe somatic mutation were $10.7 \%$ in $\mathrm{PCa}, 13.2 \%$ in metastatic PCa and not reported in metastatic mCRPC [35].

Among the most commonly altered signaling pathways in prostate cancer we have the phosphatase and tensin homolog (PTEN) tumor suppressor on chromosome 10 and the phosphoinositide 3-kinase (PI3K), about $40 \%$ of cases of PCa localized and $70 \%$ of cases of advanced disease have alterations in this pathway [45]. Normally, PTEN acts as lipid phosphatase converting phosphatidylinositol 3,4,5-trisphosphate into phosphatidylinositol 4,5-bisphosphate. This lipid phosphatase activity of PTEN suppresses the activation of the downstream oncogenic RAC-alpha serine/threonine-protein kinase (AKT) and mechanistic mammalian target of rapamycin (mTOR) signalling cascades, which have a fundamental role in controlling apoptosis, cell cycle progression, cellular proliferation, metabolism, differentiation and invasion. PTEN also has several other noncanonical functions as regulating cell adhesion and promoting chromosome stability and DNA repair through PI3K-independent function in the nucleus [47]. AR and PTEN/PI3K/AKT pathways cross-regulate each other by reciprocal feedback. Models of human and murine tumors show that inhibition of the PI3K pathway in PTEN-negative prostate cancer results in feedback signaling to the receptor tyrosine kinase HER2/HER3 leading to activation of AR. Conversely, blockade of AR results in activation of AKT through reduced levels of FKBP5 (FK506-binding protein 51) [48] impairing the stability of phosphatase PHLPP (pH domain leucine-rich repeat protein phosphatase) [49]. This bidirectional crosstalk between two critical survival pathways in prostate cancer provides the molecular rationale for simultaneously targeting both pathways [46].

\section{Clinical implications and perspectives}

Defects in DNA repair are present in a significant proportion of patients with prostate cancer. These alterations are associated with developing PCa and with developing more aggressive forms of the disease mutations affecting DDR pathway genes. Then, the knowledge about this topic can change the treatment selection resulting in a potential prognostic impact [50].

TOPARP-A was a phase II trial where 50 patients with mCRPC were treated with Olaparib, a PARP inhibitor, $400 \mathrm{mg}$ twice daily until disease progression. The primary endpoint was the composite response rate defined either as an objective response according to Response Evaluation Criteria in Solid Tumors criteria, or a $\geq 50 \%$ reduction in prostate-specific antigen (PSA), or a reduction in the circulating tumor-cell count from $\geq 5$ per $7.5 \mathrm{ml}$ of blood to 
$<5$ per $7.5 \mathrm{ml}$. All patients had prior treatment with docetaxel and 49 (98\%) with abiraterone or enzalutamide. Sixteen of 49 (33\%) evaluable patients had a response. Remarkably, however, 14 of the 16 responders had homozygous deletions, deleterious mutations, or both in DNA-repair genes - including BRCA1/2, ATM, Fanconi's anemia genes, and CHEK2 [51].

TOPARP-B, an open-label, phase II trial in which men with HRRmutated mCRPC that had progressed on at least one taxane therapy were treated with olaparib $400 \mathrm{mg}$ or $300 \mathrm{mg}$ twice daily in a randomized fashion. The primary endpoint was identical to the TOPAPRP-A trial. A targetable HRR gene aberration was found in 161 of $592(27.2 \%)$ patients who underwent a targeted next-generation tumor sequencing. However, sequencing could not be performed on 119 (17\%) of consented patients because of insufficient or poor-quality tissue. The confirmed composite response rate was $54.3 \%$ (95\% confidence interval [CI] 39.0-69.1) in the $400 \mathrm{mg}$ cohort and 39.1\% (95\% CI 25.1-54.6) in the $300 \mathrm{mg}$ cohort ( $\mathrm{p}=0.14$ ). Median radiographic progression-free survival (rPFS) was 5.5 months (95\% CI 4.4-8.3) in the 400 mg cohort and 5.6 months (3.77.7) in the $300 \mathrm{mg}$ cohort. The predefined criteria for success were met for the $400 \mathrm{mg}$ regimen but not for the $300 \mathrm{mg}$ regimen [52].

The PRO found trial was an open-label, phase III randomized (2:1) controlled trial of olaparib versus physician's choice of enzalutamide or abiraterone in men with metastatic castration resistant prostate cancer (MCRPC) with alterations in at least one of 15 homologous recombination repair genes who had disease progression on next-generation hormonal agent. Patients were randomized to olaparib (300 mg BID; n=256) or physician's choice of enzalutamide or abiraterone (control; $n=131$ ). Cohort A included 245 patients with at least one alteration in BRCA1, BRCA2, or ATM, and cohort B included 142 patients with at least one alteration in any of the other 12 prespecified genes (BARD1, BRIP1, CDK12, CHEK1, CHEK2, FANCL, PALB2, PPP2R2A, RAD51B, RAD51C, RAD51D and RAD54L). Crossover to olaparib was allowed after imaging-based disease progression. The trial met its primary endpoint of an improvement in radiographic progression-free survival with olaparib in mCRPC with alterations in BRCA1, BRCA2, or ATM (Cohort A). Key secondary endpoints, including improved overall survival in Cohort A, were also met - 19.1 months with olaparib versus 14.7 months with control therapy (HR for death, 0.69; 95\% confidence interval [CI], 0.50 to $0.97 ; \mathrm{P}=0.02$ ). In cohort $\mathrm{B}$, the median duration of overall survival was 14.1 months with olaparib and 11.5 months with control therapy (HR for death, 0.96; 95\% CI, 0.63 to 1.49). In the overall population (cohorts A and B), the corresponding durations were 17.3 months and 14.0 months (HR for death,
0.79 ; $95 \% \mathrm{CI}, 0.61$ to 1.03$)$. Overall, 86 of 131 patients $(66 \%)$ in the control group crossed over to receive olaparib (56 of 83 patients [67\%] in cohort A). A sensitivity analysis that adjusted for crossover to olaparib showed hazard ratios for death of 0.42 (95\% CI, 0.19 to 0.91 ) in cohort A, 0.83 (95\% CI, 0.11 to 5.98) in cohort $\mathrm{B}$, and 0.55 (95\% CI, 0.29 to 1.06 ) in the overall population. The most common adverse events among the patients in the olaparib group and those who crossed over to receive olaparib were anemia, nausea, and fatigue or asthenia. These results led to the approval of olaparib treatment in patients with MCRPC who had progressed on prior enzalutamide or abiraterone with homologous recombination repair alterations. Patients who had tumors with a BRCA1 or BRCA2 alteration, appeared to derive the greatest benefit from olaparib with respect to overall survival. Assessment of olaparib in patients in Cohort B with rare homologous recombination repair gene alterations provides evidence of activity, which warrants further assessment. However, PPP2R2A does not show benefit (HR for death 5.11, 95\% CI, 1.10-35.73) and preclinical studies do not support PARP inhibitor sensitivity [53].

Another PARP inhibitor that has been developed and FDA-approved for HRR-mutated (germline and/or somatic) MCRPC was rucaparib. TRITON2 [54] was a multicenter, single-arm, clinical trial of rucaparib in previously treated men with taxane-based chemotherapy and at least one novel antihormonal agent. The TRITON2 study showed an objective response rate for patients with a BRCA1/2 alterations of $43.5 \%$ (27/62 patients) when assessed by independent radiology review and $50.8 \%$ (33 of 65 patients) when assessed by the investigators, and a similar proportion achieved PSA responses as well. When HRD alterations in nonBRCA1/2 genes were analyzed [55] (ATM, CDK12, CHEK2 and other), minimal response was observed. Important toxicities that were reported include nausea, anemia, elevation in AST/ALT and gastrointestinal and hematological side effects. A confirmatory trial TRITON3 is currently ongoing. The design of this trial is like the PROfound trial, with 400 men with BRCA1/2 or ATM-mutated mCRPC randomized to rucaparib or standard of care therapy in $2: 1$ fashion. The primary endpoint is rPFS and the results are expected by 2022 .

Other two iPARP drugs are being tested for HRR-aberrant mCRPC: niraparib and talazoparib. GALAHAD [56] and TALAPRO-1 [57] are ongoing phase II trials of niraparib and talazoparib (respectively) in men with HRR-aberrant mCRPC that has progressed after at least one novel antihormonal agent and taxane-based chemotherapy. There are also phase III trials with those drugs. MAGNITUDE is a study with niraparib in combination with abiraterone for the treatment of men with MCRPC who will be evaluated for 
Clinical Implications in Understanding Prostate Cancer Biology: From Androgen Receptor to Molecular Target Therapy in Metastatic Castration Resistant Prostate Cancer

HRR status and then will be assigned to one of the 2 cohorts based on their biomarker status [58]. TALAPRO2 is a randomized, phase III trial, double-blind, comparing talazoparib plus enzalutamide vs. placebo plus enzalutamide in patients with mCRPC [59]. Both studies are currently ongoing, table 1 shows currently trials with iPARP in $\mathrm{mCRPC}$.

\begin{tabular}{|c|c|c|}
\hline Study & Status & Clinical Trials Identifier \\
\hline $\begin{array}{l}\text { CASPAR - A Phase III Trial of Enzalutamide and Rucaparib as a Novel Therapy in } \\
\text { First-Line Metastatic Castration-Resistant Prostate Cancer }\end{array}$ & Suspended & NCT04455750 \\
\hline $\begin{array}{l}\text { A Phase 3, Randomized Open-label Study of Pembrolizumab (MK-3475) } \\
\text { Plus Olaparib Versus Abiraterone Acetate or Enzalutamide in Participants } \\
\text { With Metastatic Castration-resistant Prostate Cancer (mCRPC) Who Are Unselected for } \\
\text { Homologous Recombination Repair Defects and Have Failed Prior Treatment With } \\
\text { One Next-generation Hormonal Agent (NHA) and Chemotherapy (KEYLYNK-010) }\end{array}$ & Recruiting & NCT03834519 \\
\hline $\begin{array}{l}\text { TRITON3: A Multicenter, Randomized, Open Label Phase } 3 \text { Study of Rucaparib } \\
\text { versus Physician's Choice of Therapy for patients With Metastatic Castration } \\
\text { Resistant Prostate Cancer Associated With Homologous Recombination Deficiency }\end{array}$ & Recruiting & NCT02975934 \\
\hline $\begin{array}{l}\text { A Randomised, Double-blind, Placebo-controlled, Multicentre, Phase III Study of } \\
\text { Olaparib Plus Abiraterone Relative to Placebo Plus Abiraterone as First-line Therapy } \\
\text { in Men With Metastatic Castration-resistant Prostate Cancer (PROpel Study) }\end{array}$ & $\begin{array}{l}\text { Active, not } \\
\text { recruiting }\end{array}$ & NCT03732820 \\
\hline $\begin{array}{l}\text { A Phase III, Open Label, Randomized Study to Assess the Efficacy and Safety } \\
\text { of Olaparib (Lynparza }{ }^{\mathrm{TM}} \text { ) Versus Enzalutamide or Abiraterone Acetate in Men With } \\
\text { Metastatic Castration-Resistant Prostate Cancer Who Have Failed Prior Treatment } \\
\text { With a New Hormonal Agent and Have Homologous Recombination Repair Gene } \\
\text { Mutations (PROfound) }\end{array}$ & $\begin{array}{l}\text { Active, not } \\
\text { recruiting }\end{array}$ & NCT02987543 \\
\hline $\begin{array}{l}\text { A Phase 3, Randomized, Double-blind, Placebo-controlled Study of Talazoparib With } \\
\text { Enzalutamide in Metastatic Castration-resistant Prostate Cancer (TALAPRO2) }\end{array}$ & Recruiting & NCT03395197 \\
\hline $\begin{array}{l}\text { A Phase } 3 \text { Randomized, Placebo-controlled, Double-blind Study of Niraparib in } \\
\text { Combination With Abiraterone Acetate and Prednisone Versus Abiraterone Acetate } \\
\text { and Prednisone in Subjects With Metastatic Prostate Cancer (MAGNITUDE) }\end{array}$ & Recruiting & NCT03748641 \\
\hline
\end{tabular}

Table 1: Current phase 3 clinical trials ongoing in metastatica CRPC [64].

In abiraterone-treated patients with MCRPC, tumors with PTEN loss by IHC were associated with worse outcomes [60]. Therefore, combined inhibition of the AR and PI3K-Akt-mTOR pathways may result in improved benefit for patients with mCRPC. Ipatasertib (GDC-0068) is a potent, novel, selective ATP-competitive smallmolecule inhibitor of all three isoforms of Akt [60]. In IPATential150 trial patients with $\mathrm{mCRPC}$ were randomized 1:1 to receive ipatasertib $(400 \mathrm{mg} / \mathrm{d})+$ abiraterone $(1000 \mathrm{mg} / \mathrm{d})+$ prednisone ( $5 \mathrm{mg}$ bid) or placebo + abiraterone + prednisone. Coprimary endpoints were investigator-assessed radiographic rPFS by PCWG3 criteria in patients with PTEN loss tumors by immunohistochemistry and in the overall ITT. The results, presented at ESMO 2020 [62] and updated at ASCO GU 2021 [63], demonstrated an rPFS benefit for the combination of ipatasertib with abiraterone in patients with mCRPC. This benefit was seen in the ITT population as well as when patients were stratified by PTEN expression (defined by immuno- histochemistry). In ITT population, rPFS was 19.2 months with ipatasertib and 16.6 months with placebo (HR: $0.84 ; 95 \%$ CI: 0.71 , 0.99; $\mathrm{p}=0.0431$ ). In PTEN loss patients the median rPFS was 18.5 versus 16.5 months (HR 0.77; 95\% CI 0.61,0.98; $p=0.0335$ ). Serious adverse events (AEs) occurred in $40 \%$ and $23 \%$ of ipatasertib and placebo patients, respectively; AEs leading to discontinuation of ipatasertib/placebo occurred in $21 \%$ and $5 \%$. The researchers also examined outcomes based on NGS. Of 1,104 patients, NGS was evaluable for 743 patients, and PTEN loss by NGS was evaluable in 518 patients. Overall, the agreement between the two was $85.5 \%$; among 208 patients with PTEN loss by NGS, 190 patients (91.3\%) had PTEN loss by IHC as well. Among 247 patients with PTEN loss by IHC, $76.9 \%$ had PTEN loss by NGS. The analysis found that combination ipatasertib and abiraterone was superior regarding radiographic progression-free survival in patients with PTEN loss as measured by NGS (HR 0.65, 95\% CI [0.45, 0.95]). Furthermore, 
it also offered significant benefit in the group of patients with other PIK3CA/AKT1/PTEN alterations, as determined by NGS (HR 0.63, $95 \%$ CI $[0.44,0.88])$.

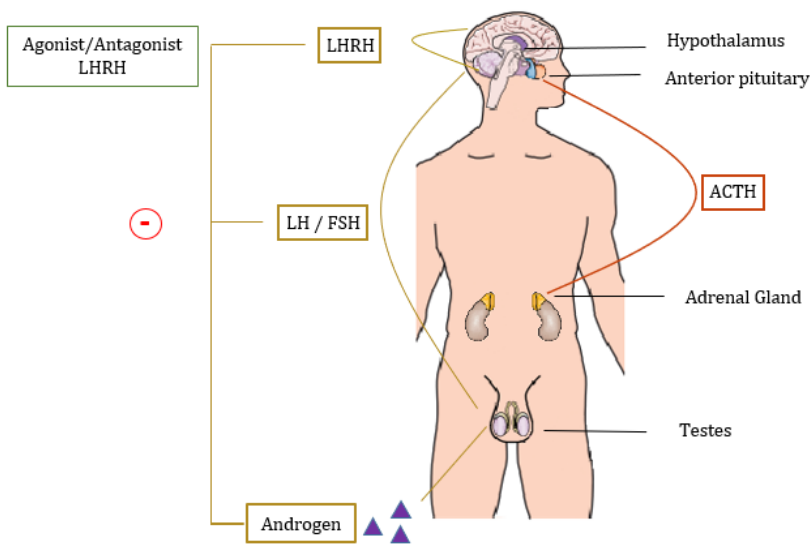

Figure 1A: The picture portrays hypothalamic-pituitary -testes-axis, the mechanism by which prostate cell proliferates by androgen binding to androgen receptor and steroid biosynthesis. It also, shows the pathways where prostate cancer treatments take place, illustrating LHRH agonist and antagonist action.

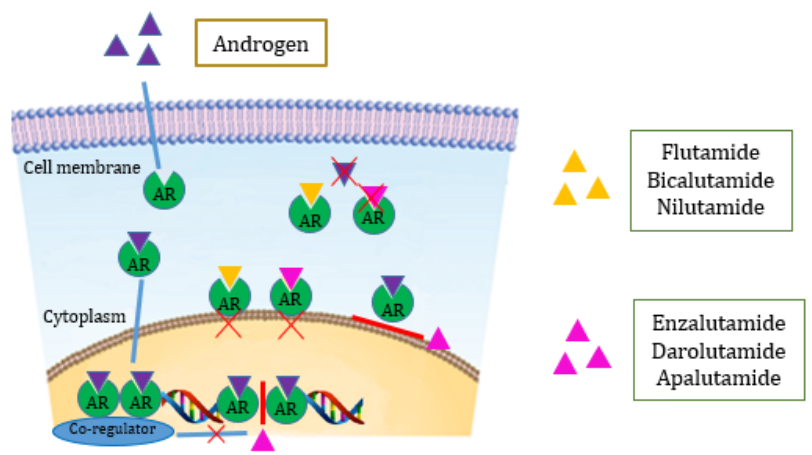

Figure 1B: The picture illustrates first-generation antiandrogen competing for androgen receptors in nucleus cytoplasm, secondgeneration antiandrogens such as enzalutamide, apalutamide and darolutamide preventing AR translocation to the nucleus.

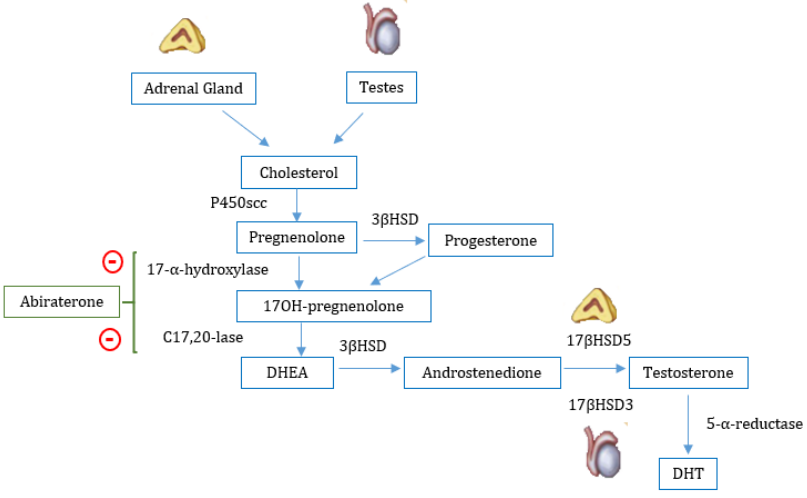

Figure 1C: The picture portrays steroid biosynthesis that takes place in adrenal gland and in the genital tissue, it also shows second-generation antiandrogens such as abiraterone acetate preventing androgen biosynthesis.

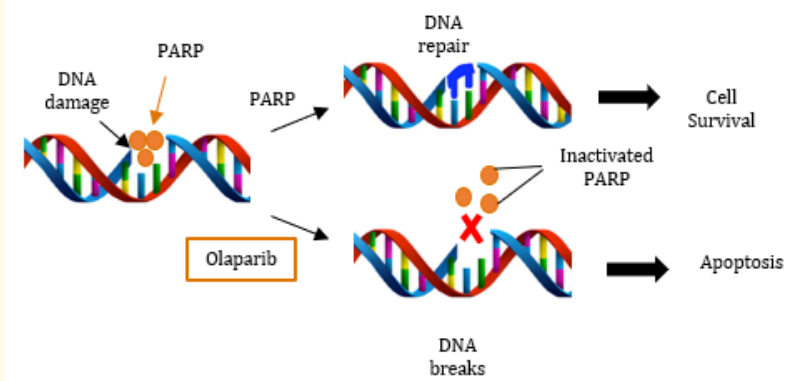

Figure 1D: The picture illustrates PARP inhibitors mechanism of action

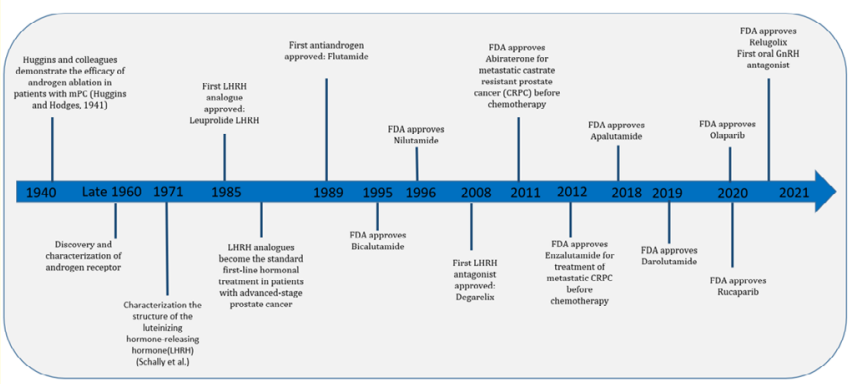

Figure 2: Timeline of the development of therapies for prostate cancer, illustrating significant events and studies for clinical applicability. 


\section{Conclusion}

Since the seminal study of Huggins [5], the field of prostate cancer has experienced a constant evolution with the development of multiple therapeutic options, as shown in figure 2. For a long time, research has focused on the androgen receptor pathway. When androgens, like testosterone and DHT bind to the AR, they induce conformational change, activation and subsequent translocation of the AR into the nucleus. Nuclear AR binds to androgen responsive elements in the DNA, resulting in transcriptional activity that can induce cellular proliferation and cellular differentiation [15]. Therefore, the blockade of the hypothalamic-pituitary-testes-axis was the target of several studies resulting in the development of agonists and antagonists of LHRH. Recently, the appeal for less invasive treatments has resulted in the development of relugolix, an oral LHRH antagonist, which has interesting PSA control rate and important reduction in cardiovascular events when compared to LHRH agonists [25]. The most comprehensive knowledge of the mechanisms of resistance of androgenic pathway such as alternative AR signaling, AR amplification, alternative splicing, intratumoral androgen production, or adrenal gland testosterone production evolved new therapeutic options such as abiraterone, enzalutamide, apalutamide and darolutamide [7-10,28].

\section{Bibliography}

1. Sung Hyuna., et al. "Global Cancer Statistics 2020: GLOBOCAN Estimates of Incidence and Mortality Worldwide for 36 Cancers in 185 Countries". CA: A Cancer Journal for Clinicians, fevereiro de (2021): 21660.

2. "Cancer Of The Prostate - Cancer Stat Facts". SEER (2021).

3. Grönberg Henrik. "Prostate Cancer Epidemiology". The Lancet 361.9360 (2003): 859-864.

4. Mateo Joaquin., et al. "DNA Repair In Prostate Cancer: Biology And Clinical Implications". European Urology 71.3 (2017): 417-425.

5. Huggins C and C V Hodges. "Studies On Prostatic Cancer: I. The Effect Of Castration, Of Estrogen And Of Androgen Injection On Serum Phosphatases In Metastatic Carcinoma Of The Prostate". CA: A Cancer Journal for Clinicians 22.4 (1972): 232-240.

6. Tannock Ian F., et al. "Docetaxel Plus Prednisone Or Mitoxantrone Plus Prednisone For Advanced Prostate Cancer". New England Journal of Medicine 351.15 (2004): 1502-1512.

7. de Bono Johann S., et al. "Abiraterone And Increased Survival In Metastatic Prostate Cancer". New England Journal of Medicine 364.21 (2011): 1995-2005.
8. Scher Howard I., et al. "Increased Survival With Enzalutamide In Prostate Cancer After Chemotherapy". New England Journal of Medicine 367.13 (2012): 1187-1197.

9. Chi Kim N., et al. "Apalutamide For Metastatic, Castration-Sensitive Prostate Cancer". New England Journal of Medicine 381.1 (2019): 13-24.

10. Fizazi Karim., et al. "Nonmetastatic, Castration-Resistant Prostate Cancer And Survival With Darolutamide". New England Journal of Medicine 383.11 (2020): 1040-1049.

11. Oefelein Michael G., et al. "Reassessment Of The Definition Of Castrate Levels Of Testosterone: Implications For Clinical Decision Making". Urology 56.6 (2000): 1021-1024.

12. SIITERI PENTTI K and JEAN D WILSON. "Testosterone Formation And Metabolism During Male Sexual Differentiation In The Human Embryo". The Journal of Clinical Endocrinology and Metabolism 38.1 (1974): 113-125.

13. Cunha Gerald R., et al. "Role Of Stromal-Epithelial Interactions In Hormonal Responses". Archives of Histology and Cytology 67.5 (2004): 417-434.

14. Deslypere JP., et al. “Testosterone And 5A-Dihydrotestosterone Interact Differently With The Androgen Receptor To Enhance Transcription Of The MMTV-CAT Reporter Gene". Molecular and Cellular Endocrinology 88.1-3 (1992): 15-22.

15. Mostaghel Elahe A., et al. "Intraprostatic Androgens And Androgen-Regulated Gene Expression Persist After Testosterone Suppression: Therapeutic Implications For Castration-Resistant Prostate Cancer". Cancer Research 67.10 (2007): 50335041.

16. Limonta Patrizia., et al. "Gnrh In The Human Female Reproductive Axis". Vitamins and Hormones (2018): 27-66.

17. Munro Neville A and MJ O'hare. "Histopathology Of The Human Adrenal Cortex". Clinics in Endocrinology and Metabolism 14.4 (1985): 791-820

18. Miller Walter L. "MECHANISMS IN ENDOCRINOLOGY: Rare Defects In Adrenal Steroidogenesis". European Journal of Endocrinology 179.3 (2018): R125-R141.

19. Parker Keith L and Bernard P Schimmer. "Transcriptional Regulation Of The Genes Encoding The Cytochrome P-450 Steroid Hydroxylases". Vitamins and Hormones (1995): 339-370.

20. Sanderson J Thomas. "The Steroid Hormone Biosynthesis Pathway As A Target For Endocrine-Disrupting Chemicals". Toxicological Sciences 94.1 (2006): 3-21. 
21. Sandow Jürgen., et al. "Pituitary Gonadotropin Inhibition By A Highly Active Analog Of Luteinizing Hormone-Releasing Hormone". Fertility and Sterility 30.2 (1978): 205-209.

22. Gründker Carsten and Günter Emons. "The Role Of Gonadotropin-Releasing Hormone In Cancer Cell Proliferation And Metastasis". Frontiers in Endocrinology 8 (2017).

23. Thompson Ian M. "Flare Associated With LHRH-Agonist Therapy". Reviews in Urology 3.3 (2001): S10-14.

24. Klotz Laurence., et al. "The Efficacy And Safety Of Degarelix: A 12-Month, Comparative, Randomized, Open-Label, ParallelGroup Phase III Study In Patients With Prostate Cancer". BJU International 102.11 (2008): 1531-1538.

25. Shore Neal D., et al. "Oral Relugolix For Androgen-Deprivation Therapy In Advanced Prostate Cancer". New England Journal of Medicine 382.23 (2020): 2187-2196.

26. Crona DJ., et al. "Androgen Receptor Targeting Drugs In Castration-Resistant Prostate Cancer And Mechanisms Of Resistance". Clinical Pharmacology and Therapeutics 98.6 (2015): 582-589.

27. Heidenreich Axel., et al. "EAU Guidelines On Prostate Cancer. Part II: Treatment Of Advanced, Relapsing, And CastrationResistant Prostate Cancer". European Urology 65.2 (2014): 467-479.

28. Knudsen Karen E and William Kevin Kelly. "Outsmarting Androgen Receptor: Creative Approaches For Targeting Aberrant Androgen Signaling In Advanced Prostate Cancer". Expert Review of Endocrinology and Metabolism 6.3 (2011): 483-493.

29. Clarke Noel., et al. "Olaparib Combined With Abiraterone In Patients With Metastatic Castration-Resistant Prostate Cancer: A Randomised, Double-Blind, Placebo-Controlled, Phase 2 Trial”. The Lancet Oncology 19.7 (2018): 975-986.

30. Teply Benjamin A and Emmanuel S Antonarakis. "Treatment Strategies For DNA Repair-Deficient Prostate Cancer". Expert Review Of Clinical Pharmacology 10.8 (2017): 889-898.

31. Asim Mohammad., et al. "Synthetic Lethality Between Androgen Receptor Signalling And The PARP Pathway In Prostate Cancer". Nature Communications 8.1 (2017).

32. Hakem Razqallah. "DNA-Damage Repair; The Good, The Bad, And The Ugly". The EMBO Journal 27.4 (2008): 589-605.

33. Branzei, Dana, and Marco Foiani. "Regulation Of DNA Repair Throughout The Cell Cycle". Nature Reviews Molecular Cell Biology 9.4 (2008): 297-308.
34. Chatterjee Nimrat and Graham C Walker. "Mechanisms Of DNA Damage, Repair, And Mutagenesis". Environmental And Molecular Mutagenesis 58.5 (2017): 235-263.

35. Lang Shona., et al. "A Systematic Review Of The Prevalence Of DNA Damage Response Gene Mutations In Prostate Cancer". International Journal of Oncology (2019).

36. Pritchard Colin C., et al. "Inherited DNA-Repair Gene Mutations In Men With Metastatic Prostate Cancer". New England Journal of Medicine 375.5 (2016): 443-453.

37. Hanahan Douglas and Robert A Weinberg. "Hallmarks Of Cancer: The Next Generation". Cell 144.5 (2011): 646-674.

38. Burdak-Rothkamm Susanne., et al. "DNA Damage Repair Deficiency In Prostate Cancer". Trends in Cancer 6.11 (2020): 974 984.

39. Dall'Era Marc A., et al. "Germline And Somatic DNA Repair Gene Alterations In Prostate Cancer". Cancer 126.13 (2020): 2980-2985.

40. Nguyen Luan., et al. "Pan-Cancer Landscape Of Homologous Recombination Deficiency". Nature Communications 11.1 (2020).

41. Fridlich Ram., et al. "BRCA1 And BRCA2 Protect Against Oxidative DNA Damage Converted Into Double-Strand Breaks During DNA Replication". DNA Repair 30 (2015): 11-20.

42. Bishop Alexander JR and Robert H Schiestl. "Homologous Recombination And Its Role In Carcinogenesis". Journal Of Biomedicine And Biotechnology 2.2 (2002): 75-85.

43. Wei, Yu., et al. "Germline DNA Repair Gene Mutation Landscape In Chinese Prostate Cancer Patients". European Urology 76.3 (2019): 280-283.

44. Fradet-Turcotte, Amélie., et al. "BRCA2 Functions: From DNA Repair To Replication Fork Stabilization". Endocrine-Related Cancer 23.10 (2016): T1-T17.

45. Marshall Catherine Handy., et al. "Prevalence Of DNA Repair Gene Mutations In Localized Prostate Cancer According To Clinical And Pathologic Features: Association Of Gleason Score And Tumor Stage". Prostate Cancer and Prostatic Diseases 22.1 (2018): 59-65.

46. Carver Brett S., et al. "Reciprocal Feedback Regulation Of PI3K And Androgen Receptor Signaling In PTEN-Deficient Prostate Cancer". Cancer Cell 19.5 (2011): 575-586.

47. Jamaspishvili Tamara., et al. "Clinical Implications Of PTEN Loss In Prostate Cancer". Nature Reviews Urology 15.4 (2018): 222-234. 
48. Pei Huadong., et al. "FKBP51 Affects Cancer Cell Response To Chemotherapy By Negatively Regulating Akt". Cancer Cell 16.3 (2009): 259-266.

49. Gao Tianyan., et al. "PHLPP: A Phosphatase That Directly Dephosphorylates Akt, Promotes Apoptosis, And Suppresses Tumor Growth". Molecular Cell 18.1 (2005): 13-24.

50. Lozano Rebeca., et al. "Genetic Aberrations In DNA Repair Pathways: A Cornerstone Of Precision Oncology In Prostate Cancer". British Journal of Cancer 124.3 (2020): 552-563.

51. Mateo Joaquin., et al. "DNA-Repair Defects And Olaparib In Metastatic Prostate Cancer". New England Journal of Medicine 373.18 (2015): 1697-1708.

52. Mateo Joaquin., et al. "Olaparib In Patients With Metastatic Castration-Resistant Prostate Cancer With DNA Repair Gene Aberrations (TOPARP-B): A Multicentre, Open-Label, Randomised, Phase 2 Trial". The Lancet Oncology 21.1 (2020): 162-174.

53. Hussain Maha., et al. "Survival With Olaparib In Metastatic Castration-Resistant Prostate Cancer". New England Journal of Medicine 383.24 (2020): 2345-2357.

54. Abida W., et al. "Preliminary Results From The TRITON2 Study Of Rucaparib In Patients (Pts) With DNA Damage Repair (DDR)-Deficient Metastatic Castration-Resistant Prostate Cancer (Mcrpc): Updated Analyses". Annals of Oncology 30 (2019): 327-v328.

55. Abida Wassim., et al. "Non-BRCA DNA Damage Repair Gene Alterations And Response To The PARP Inhibitor Rucaparib In Metastatic Castration-Resistant Prostate Cancer: Analysis From The Phase II TRITON2 Study". Clinical Cancer Research 26.11 (2020): 2487-2496.

56. Smith Matthew Raymond., et al. "Phase II Study Of Niraparib In Patients With Metastatic Castration-Resistant Prostate Cancer (Mcrpc) And Biallelic DNA-Repair Gene Defects (DRD): Preliminary Results Of GALAHAD... Journal of Clinical Oncology 37.7 (2019): 202-202.

57. De Bono Johann S., et al. "TALAPRO-1: A Phase II Study Of Talazoparib (TALA) In Men With DNA Damage Repair Mutations (Ddrmut) And Metastatic Castration-Resistant Prostate Cancer (Mcrpc)-First Interim Analysis (IA)... Journal of Clinical Oncology 38.6_suppl (2020): 119-119.
58. "A Study Of Niraparib In Combination With Abiraterone Acetate And Prednisone Versus Abiraterone Acetate And Prednisone For Treatment Of Participants With Metastatic Prostate Cancer - Full Text View - Clinicaltrials.Gov". Clinicaltrials.Gov (2021).

59. "Talazoparib + Enzalutamide Vs. Enzalutamide Monotherapy In Mcrpc - Full Text View - Clinicaltrials.Gov". Clinicaltrials.Gov (2021).

60. Ferraldeschi Roberta., et al. "PTEN Protein Loss And Clinical Outcome From Castration-Resistant Prostate Cancer Treated With Abiraterone Acetate". European Urology 67.4 (2015): 795-802.

61. Lin Jie., et al. "Targeting Activated Akt With GDC-0068, A Novel Selective Akt Inhibitor That Is Efficacious In Multiple Tumor Models". Clinical Cancer Research 19.7 (2013): 1760-1772.

62. de Bono JS., et al. "LBA4 Ipatential150: Phase III Study Of Ipatasertib (Ipat) Plus Abiraterone (Abi) Vs Placebo (Pbo) Plus Abi In Metastatic Castration-Resistant Prostate Cancer (Mcrpc)". Annals of Oncology 31 (2020): S1153-S1154.

63. De Bono Johann S., et al. "PI3K/AKT Pathway Biomarkers Analysis From The Phase III Ipatential150 Trial Of Ipatasertib Plus Abiraterone In Metastatic Castration-Resistant Prostate Cancer". Journal of Clinical Oncology 39.6 (2021): 13-13.

64. "Home - Clinicaltrials.Gov". Clinicaltrials.Gov (2021).

Volume 5 Issue 6 June 2021

(C) All rights are reserved by Eliza Dalsasso Ricardo. 